Reprod. Nutr. Dévelop., 1988, 28 (1), 119-120.

\title{
Digestion ruminale comparée du son et de la pulpe de betteraves chez le mouton et la vache
}

\author{
M. DOREAU, Brigitte MICHALET-DOREAU $(*)$, C. PONCET $\left({ }^{* *}\right)$, Marie-Pierre LE GUEN \\ Laboratoire de la Lactation \\ (*) Laboratoire des Aliments \\ (**) Laboratoire de la Digestion \\ I.N.R.A., Theix, 63122 Ceyrat, France.
}

Summary. Ruminal digestion was compared in cows and sheep receiving ad libitum $40 \%$ hay and $60 \%$ wheat bran or beet pulp. Organic matter (OM) digestibility was $5 \%$ higher for pulp diet, but ruminal OM digestibility was the same for both diets. Ruminal retention time was shorter for pulp, but its rate of digestion appeared to be more rapid. Hay ruminal retention time was higher in cows, but ruminal digestibility was not.

La digestibilité et le temps de séjour des rations riches en aliments concentrés dans le tube digestif du ruminant varient avec la nature de ceux-ci, ainsi qu'avec l'espèce anirnale (Michalet-Doreau et al., 1987 ; Poncet et al., 1987). Les résultats présentés ici, obtenus dans le cadre de ces deux essais, ont permis de préciser la part du rumen dans les variations observées.

Matériel et méthodes. Deux régimes comprenant $40 \%$ de foin (luzernedactyle) et $60 \%$ de son de blé (ration S : $21,1 \%$ de cellulose brute (CB) et 2,30\% d'azote (N), ou de pulpe de betteraves déshydratées (plus urée : $1 \%$ de la MS) (ration $P: 24,8 \% C B$ et $2,30 \% N$ ) ont été offerts à volonté à 2 vaches laitières en production (poids vif ( $\mathrm{PV}$ ) : $557 \mathrm{~kg}$ ) et à 2 moutons castrés ( $\mathrm{PV}: 56 \mathrm{~kg}$ ) munis de canules du rumen et du duodénum proximal, en 2 distributions égales espacées de 12 heures. Les proportions des deux aliments étaient maintenues par réajustement quotidien de l'aliment le moins bien consommé.

Les digestibilités de la matière organique (MO) et de la $\mathrm{CB}$ ont été mesurées par collecte totale des fèces durant 6 jours, celles de la MO et de l'azote dans le rumen à partir du flux duodénal, en utilisant comme marqueurs de l'oxyde de chrome, du polyéthylène glycol (PEG) et du Co-EDTA chez les vaches et du PEG chez les moutons. Le pH, la teneur en ammoniac et en acides gras volatils (AGV) et la composition du mélange des AGV ont été mesurés sur des échantillons de liquide de rumen prélevés 30 min avant et $3 \mathrm{~h}$ après la distribution d'aliments du matin, le taux de renouvellement du liquide du rumen par la décroissance de concentration ruminale en PEG (vaches et moutons) et en Co-EDTA (vaches) pendant $30 \mathrm{~h}$. Le temps de séjour du foin et de l'aliment concentré dans le rumen ont été mesurés par introduction d'aliments marqués spécifiquement : foin (vaches : Ytterbium ; moutons : ${ }^{169}$ Ytterbium) et concentré (vaches : Europium ; moutons : ${ }^{141}$ Cérium) selon la technique de trempage et fixation sélective mise au point par Poncet (non publié), puis prélèvements au niveau duodénal échelonnés sur $72 \mathrm{~h}$.

Résultats et discussion. La digestion plus élevée du régime $\mathrm{P}$ semble due à un accroissement de la digestion dans l'intestin. La digestion ruminale de la pulpe est 
peut-être limitée par un temps de séjour plus court que celui du son, malgré une vitesse de digestion plus rapide, comme en témoigne la teneur plus élevée en AGV (tabl. 2). L'augmentation de la teneur en propionate après le repas est modérée et identique pour les deux régimes. Le flux duodénal d'azote non ammoniacal est le même pour les rations $S$ et $P$, lorsqu'il est rapporté à la quantité de MO apparemment digérée dans le rumen $(48,3$ et $46,9 \mathrm{~g} / \mathrm{kg})$ ou d'azote ingéré $(0,93$ et 0,93$)$. Les teneurs en ammoniac du rumen sont cependant très supérieures avant comme après le repas pour le régime $S$.

TABL. 1. - Digestibilités totale et ruminale, temps de séjour dans le rumen. Effets espèce, régime, interaction : $E, R, I(1 \%), e, r, i(5 \%)$.

\begin{tabular}{|c|c|c|c|c|c|}
\hline & \multicolumn{2}{|c|}{ Régime $\mathrm{S}$} & \multicolumn{2}{|c|}{ Régime $P$} & \multirow{2}{*}{$\begin{array}{l}\text { Différences } \\
\text { significatives }\end{array}$} \\
\hline & Moutons & Vaches & Moutons & Vaches & \\
\hline Quantité ingérées (g MS/kg poids métabolique) & 70,7 & 125,8 & 96,3 & 148,1 & - \\
\hline \multirow{2}{*}{$\begin{aligned} \text { Digestibilité }(\%) & \text { - Matière organique } \\
& \text { - Cellulose brute }\end{aligned}$} & 63,7 & 66,2 & 70,4 & 71,6 & $r$ \\
\hline & 50,8 & 50,4 & 59,6 & 64,7 & $\mathrm{R}$ \\
\hline \multirow{2}{*}{$\begin{array}{l}\text { Digestibilité ruminale ( } \% \text { ingéré). Matière organique } \\
\text { Taux de renouvellement du liquide du rumen }(\% / \mathrm{h})\end{array}$} & 53,1 & 48,2 & 53,2 & 51,7 & e \\
\hline & 13,3 & 12,8 & 14,7 & 12,2 & \\
\hline \multirow{2}{*}{$\begin{aligned} \text { Temps de séjour dans le rumen }(\mathrm{h}) & - \text { Foin } \\
& - \text { Concentré }\end{aligned}$} & 19,6 & 27,0 & 17,5 & 25,6 & $E$ \\
\hline & 23,3 & 20,8 & 14,3 & 16,4 & $\bar{R}$ \\
\hline
\end{tabular}

TABL. 2. $-p H$ et produits terminaux de la digestion ruminale. AV. R. : avant repas. - AP. R. : après repas. Effets statistiques : cf. tabl. 1.

\begin{tabular}{|c|c|c|c|c|c|c|c|c|c|c|}
\hline & \multicolumn{4}{|c|}{ Régime $\mathrm{S}$} & \multicolumn{4}{|c|}{ Régime $P$} & \multirow{2}{*}{\multicolumn{2}{|c|}{$\begin{array}{l}\text { Différences } \\
\text { significatives }\end{array}$}} \\
\hline & \multicolumn{2}{|c|}{ Moutons } & \multicolumn{2}{|c|}{ Vaches } & \multicolumn{2}{|c|}{ Moutons } & \multicolumn{2}{|c|}{ Vaches } & & \\
\hline & AV. R. & AP. R. & AV. R. & AP. R. & AV. R. & AP. R. & AV. R. & AP. R. & AV. R. & AP. R. \\
\hline $\mathrm{pH}$ & 6,50 & 6,26 & 6,85 & 6,81 & 6,60 & 5,72 & 6,87 & 6,40 & $\mathrm{e}$ & $\mathrm{Er}$ \\
\hline AGV (Millimoles/I) & 54,3 & 65,8 & 83,1 & 95,8 & 69,6 & 109,7 & 89,5 & 121,2 & $\mathrm{e}$ & $\mathrm{R}$ \\
\hline Acétate ( $\%$ molaire) & 66,5 & 64,7 & 66,7 & 65,9 & 63,0 & 67,4 & 68,7 & 68,4 & & \\
\hline Propionate ( $\%$ mol.) & 15,8 & 19,6 & 15,9 & 17,4 & 20,2 & 20,7 & 17,0 & 19,2 & e $R i$ & \\
\hline Butyrate $(\%$ mol.) & 13,1 & 11,5 & 13,4 & 12,5 & 13,6 & 10,3 & 11,9 & 10,8 & & \\
\hline Ammoniac $\{m g / l\}$ & 183 & 241 & 158 & 226 & 88 & 146 & 71 & 154 & $\mathrm{R}$ & $\mathrm{R}$ \\
\hline
\end{tabular}

Les différences entre moutons et vaches ne peuvent être toutes clairement expliquées à l'aide des paramètres mesurés. Ainsi, le $\mathrm{pH}$ est systématiquement plus élevé chez les vaches, la proportion de propionate restant toutefois comparable. Le taux de renouvellement du liquide est très élevé dans cet essai pour les moutons. La part du rumen dans la digestion de la MO est plus faible $(P<0,01)$ chez les vaches $(72,5 \%)$ que chez les moutons $(79,5 \%)$, malgré un temps de séjour du foin plus long, comme dans les essais de Uden et al. (1982) et de Prigge et al. (1984), et une concentration en AGV plus élevée. Une mastication plus efficace chez le mouton pourrait expliquer ces résultats.

Michalet-Doreau B., Geay Y., Doreau M., Poncet C., 1987. Reprod. Nutr. Dévelop., 27, $227-228$. Poncet C., Gomez L., Michalet-Doreau B., Geay Y., 1987. Reprod. Nutr. Dévelop., 27, $219-220$. Prigge E. C., Baker M. J., Varga G. A., 1984. J. Anim. Sci., 58, 213-221.

Uden P., Rounsaville T. R., Wiggans G. R., Van Soest P. J., 1982. Br. J. Nutr., 48, 329-339. 